\title{
Caracterización de estados de tiempo en el suroeste bonaerense, Argentina ${ }^{1}$
}

\author{
María Belén Ramos², Alicia M. Campo ${ }^{3}$
}

\begin{abstract}
RESUMEN
Se analizan los estados de tiempo para el Suroeste bonaerense argentino considerando el método analítico y el sintético como complementarios. Se centra el estudio en la ciudad de Punta Alta donde se localiza una estación meteorológica perteneciente al grupo de trabajo y que registra información desde el año 2000. El objetivo general es analizar y caracterizar los estados de tiempo predominantes en el Suroeste bonaerense. Para ello se emplearon las cartas del tiempo proporcionadas por el Servicio Meteorológico Nacional y los datos de la estación meteorológica situada en el centro de la ciudad de Punta Alta. Se elaboró una clasificación de estados de tiempo típicos en el área basada en elementos principales: temperatura, presión y precipitación. Esta categorización permitió determinar los porcentajes de frecuencia de cada tipo de tiempo.
\end{abstract}

Palabras clave: Estados de tiempo, Suroeste bonaerense, carta del tiempo.

\begin{abstract}
This study analyzes weather conditions in the Southwest of the Buenos Aires Province, Argentine, considering analytical and synthetically methods complementarily. The study is centered in the city of Punta Alta where a meteorological station is located. The data information is from the year 2000. The general objective is to analyze and characterize the predominant weather conditions in the Southwest of Buenos Aires Province. Weather Charts provided by the National Meteorological Service were used. A classification of typical weather states in the area was made according to principal climatic elements: pressure, temperature and precipitations. This categorization allowed for the determining of average frequency of each type of weather.
\end{abstract}

Key words: Weather conditions, Southwest of the province of Buenos Aires, weather Charts.

Estudiar el clima de un lugar requiere en primera instancia determinar la escala de estudio, la cual varía de acuerdo con la extensión del espacio considerado. Los estudios climáticos regionales se basan en la circula-

\footnotetext{
1 Este artículo corresponde al Proyecto "La Geografía Física del Sur de la provincia de Buenos Aires. Relaciones del hombre y el medio natural", que cuenta con el apoyo de la SGCYT. Universidad Nacional del Sur, Bahía Blanca. Artículo recibido el 9 de mayo de 2007 y aceptado el 6 de marzo de 2008.
}

ción celular y en sus relaciones con los factores geográficos, ellos encierran diferenciaciones que dan lugar a unidades menores de investigación, los climas locales. El clima

\footnotetext{
2 Comisión de Investigaciones Científicas de la provincia de Buenos Aires (CIC) (Argentina) Depto. de Geografía y Turismo. Universidad Nacional del Sur, Bahía Blanca (Argentina).E-mail: mberamos@uns.edu.ar

3 Consejo Nacional de Investigaciones Científicas y Técnicas (CONICET) (Argentina) - Depto. de Geografía y Turismo. Universidad Nacional del Sur, Bahía Blanca (Argentina).E-mail: amcampo@uns.edu.ar
} 
local constituye una de las unidades de análisis más pequeñas en la escala de los estudios climáticos. Representa una individualidad del clima regional alterado por condiciones topográficas, proximidad del mar, presencia de masas forestales o intervención del hombre. A esta escala es posible medir cambios en períodos de tiempo relativamente breves de modo que se puede investigar en forma directa la dinámica de los procesos (Capelli et al., 2005: 16). El aspecto diario que presenta la atmósfera y que el hombre percibe es el "tiempo atmosféri-

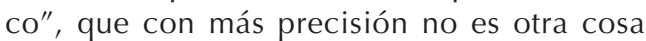
que el estado físico de la atmósfera en cada instante, en un lugar determinado, y su evolución en un tiempo relativamente corto. El "tiempo" está continuamente cambiando, la atmósfera tiene cada día una fisonomía distinta, en la variabilidad cotidiana del "tiempo" se encuentran inmersas la mayor parte de las actividades humanas, pero hay algo permanente en el conjunto innumerable de estados de "tiempo" que se vienen dando en una determinada región o área geográfica, que asigna a esta unas características concretas, que permiten diferenciar unas regiones de otras, y a lo permanente del tiempo lo Ilamamos "clima" (Almarza, 2005). Son de gran relevancia los estudios de estados de tiempo realizados por geógrafos y según Rey Benadero (2001: 198), los conocimientos cartográficos y de dinámica atmosférica del geógrafo lo convierten en el técnico ideal para evaluar eficientemente la situación atmosférica: relato de los principales actores atmosféricos del episodio (masas de aire, barreras orográficas, fuentes de humedad) y su rol en la situación atmosférica tratada. Por otra parte, el mismo autor resalta la importancia para el análisis de la situación atmosférica de la climatología sinóptica y analítica.

La República Argentina se localiza en la porción más austral de América del Sur, está bajo la influencia de diferentes masas de aire que por su desplazamiento estacional definen las características climáticas del continente $y$, en particular, del Suroeste de la provincia de Buenos Aires (Campo de Ferreras et al., 2004: 7). El área de estudio se ve afectada por la irrupción o interacción de masas de aire de distintas características y origen hecho distintivo de la zona planetaria de los climas templados. Las masas de aire en cuestión son: Aire Antártico, que se genera en la región cubierta de hielo y nieve, es fría, seca y estable; Aire Polar continental, cuyo origen es la región continental subpolar, es fría y seca; Aire Polar marítimo, proviene de la zona subpolar, es fría y húmeda; Aire Tropical continental, se genera en la zona continental subtropical de altas presiones, es cálida y seca y Aire Tropical marítimo, proveniente de los anticiclones subtropicales, sobre los océanos, es cálida y húmeda (Servicio Meteorológico Nacional, 1987: 1).

No obstante, la influencia e intensidad de las masas de aire varían según las estaciones del año, los centros de acción acompañan el movimiento aparente del Sol dando como resultado el desplazamiento de todo el sistema hacia el Norte en la estación invernal y hacia el Sur en la estival. En verano las masas Tropicales continentales se ven restringidas al interior del continente que se encuentra muy caliente y ocasionan sequía y pérdida de energía por enfriamiento nocturno. Por otra parte, las masas de aire Tropicales marítimas, que surgen del borde occidental del Anticiclón del Atlántico Sur, ingresan al Sur de la provincia de Buenos Aires en sentido Norte Sur. Si bien a estas latitudes ya han perdido gran parte de sus propiedades iniciales igualmente son generadoras de lluvias y de las típicas tormentas de verano que se producen en la región (Campo de Ferreras et al., 2004: 9).

En invierno la zona de las Altas Presiones Subtropicales se desplaza hacia latitudes más bajas y, a su vez, el sistema de las Bajas Presiones Subpolares hace sentir más su influencia en la Patagonia acompañando el avance de los frentes fríos hasta la región Chaco Pampeana. Las masas de aire Polares continentales aumentan su extensión en el invierno y el aire frío es muy estable debido al enfriamiento de la Patagonia. En la región Pampeana y en particular en el Sur de la provincia de Buenos Aires se destaca la influencia de aire frío y húmedo que en general proviene de desprendimientos celulares de las Altas Presiones del Pacífico Sur. Los mismos se ubican en el Océano Atlántico frente a las costas bonaerenses de acuerdo a su trayectoria de ingreso al continente con 
sentido Oeste - Este. Por su giro retrógrado trae a la región Pampeana aire procedente del mar generando varios días de mal tiempo lluvioso y ventoso. Las masas de aire Tropicales continentales intensifican en invierno su área de dominio, mientras que las Tropicales marítimas se desplaza hacia el Norte y no se hace sentir en el territorio argentino.

Para caracterizar el clima del Suroeste bonaerense es fundamental, según Pedelaborde (1970), comenzar por el estudio de la sucesión de estados de tiempo. En este contexto, se analizaron en este estudio los datos de los estados atmosféricos referidos a situaciones sinópticas típicas que conforman al clima local. Son numerosas las publicaciones internacionales que abordan el estudio de características climáticas zonales, regionales y locales mediante el análisis de los datos del tiempo (Lam et al., 2005; Olgyay, 1992; Barth \& Steinkohl, 2004). Abaurrea et al. (s/f.) han realizado análisis de situaciones sinópticas en los episodios de ola de calor en el centro del valle del Ebro; Rivera (2003) investigó las situaciones de Iluvias torrenciales en el área mediterránea española y Caviedes (1969) analizó los estados de tiempo típicos de Valparaíso, Chile Central.

En el área de estudio solo se han efectuado investigaciones de estados de tiempo específicos relacionados con la salud de la población de la ciudad de Punta Alta. Este hecho se debió a que la ciudad no poseía datos meteorológicos propios hasta el mes de abril de 2000, fecha en que se colocó la primera estación meteorológica perteneciente al Departamento de Geografía y Turismo de la Universidad Nacional del Sur.

La ciudad de Punta Alta, cabecera del partido de Coronel de Marina Leonardo Rosales, se localiza a $38^{\circ} 43^{\prime}$ y $62^{\circ} 06^{\prime}$, en el Suroeste de la provincia de Buenos Aires. Limita al Oeste y Noroeste con el partido de Bahía Blanca, al Norte con el partido de Co-

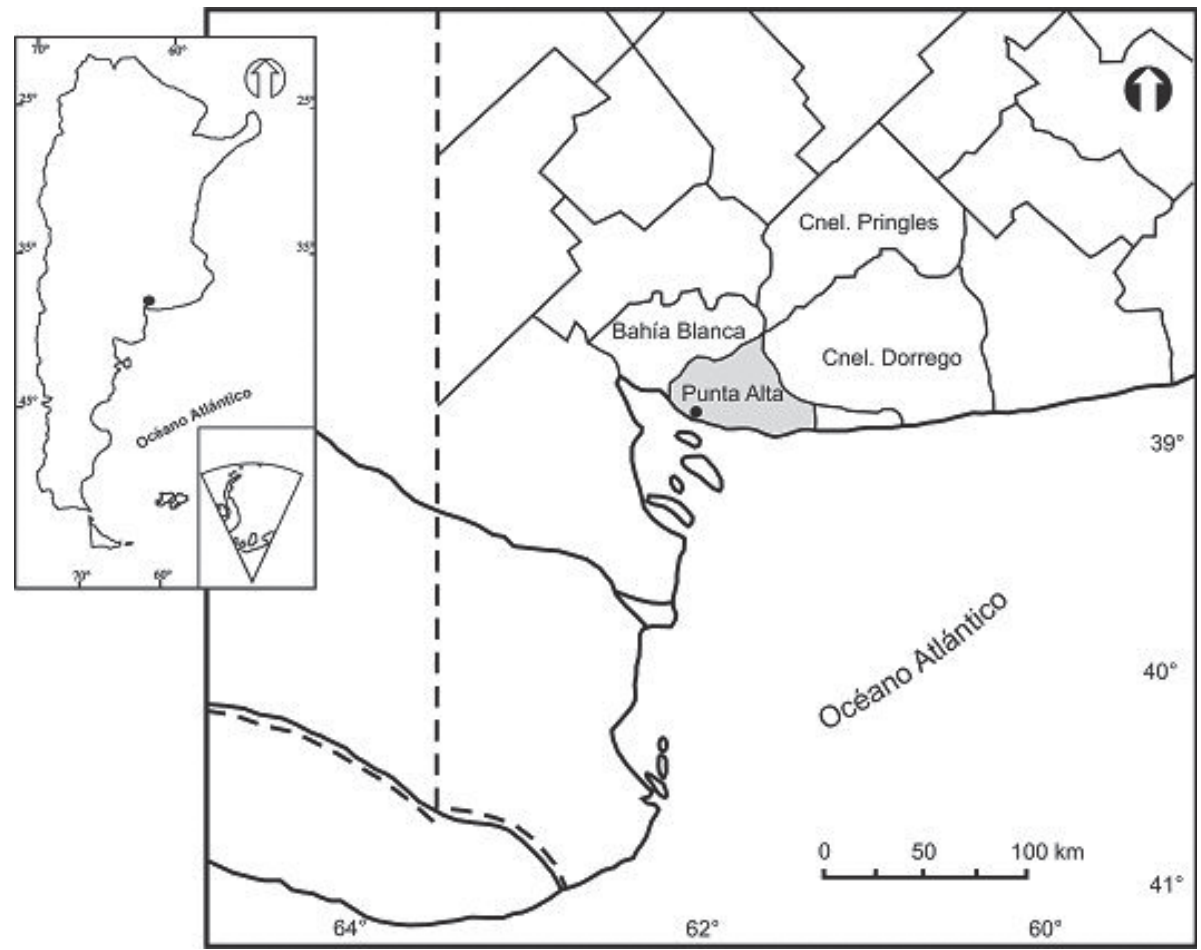

Fuente: Elaboración propia. 
ronel Pringles, al Sur con el Océano Atlántico y al Sureste con el partido de Coronel Dorrego, cuyo límite natural es el río Sauce Grande (Figura $N^{\circ} 1$ ). Dista 27 km de la ciudad de Bahía Blanca y 673 km de la Capital Federal. Por su proximidad al mar presenta suelos salinos y arenosos con vegetación típica asociada a ellos.

\section{Objetivos de estudio}

Los objetivos del estudio consisten en analizar y caracterizar los estados de tiempo predominantes en el Suroeste bonaerense y en particular para la ciudad de Punta Alta. A tal fin se analizaron las situaciones atmosféricas que los producen, la posición de los centros de acción y masas de aire. Por otra parte, se determinó la frecuencia de aparición de los diferentes estados de tiempo y el porcentaje de días con respecto al total considerado.

\section{Métodos y materiales}

El estudio de los estados de tiempo se plantea considerando el método analítico y el sintético como complementarios. Es importante resaltar que no existe a nivel internacional unificación en cuanto a una metodología de clasificación de los tipos de tiempo, sino que a partir de las características que presenta cada área y los objetivos específicos de estudio, se adopta la que se considera más conveniente. Font Tullot (2000), Martín Vide (1991), Martin Vide y Olcina Cantos (2001) son algunos de los autores que han elaborado catálogos y clasificaciones de situaciones sinópticas y tiempos asociados. No obstante, el método aplicado en este trabajo es el de Pejenaute Goñi (1990: 36), quien establece una doble clasificación diaria: según los tipos de tiempo y según las situaciones atmosféricas.

Se realizó el análisis estadístico estándar de los datos de la estación meteorológica situada en el centro de la ciudad de Punta Alta. Desde el método analítico se ha identificado cada uno de los días durante seis años (2000 - 2005) de acuerdo a tres elementos climáticos: presión, temperatura y precipitación. A partir de la combinación de dichas variables se elaboró una clasificación de estados de tiempo típicos en el área de la ciudad de Punta Alta. Esta categorización permitió determinar los porcentajes de frecuencia de cada tipo de tiempo. Cabe destacar que en el siguiente trabajo se presentan los valores generales correspondientes a los cinco años y se analiza estacionalmente el año 2003 debido a que se contada con mayor información cartográfica sinóptica diaria.

La presión fue la primera variable climática abordada; se obtuvo un valor medio a partir de los 48 registros diarios. Posteriormente, se calculó la presión media de todo el período y se efectuó una doble clasificación: tiempos ciclónicos (C) (aquellos cuya presión es inferior a la media) y tiempos anticiclónicos (A) (cuando es superior a la media).

En cuanto a la segunda variable, la temperatura, el método aplicado consideró la máxima temperatura diaria, obteniéndose la siguiente clasificación: días muy fríos (MF), con máxima menor o igual a $5{ }^{\circ} \mathrm{C}$; días fríos $(\mathrm{F})$, con máximas mayores a $5{ }^{\circ} \mathrm{C}$ o menores que $10{ }^{\circ} \mathrm{C}$; días templados $(\mathrm{T})$, con temperaturas máximas mayores o iguales a $10{ }^{\circ} \mathrm{C}$ y menores o iguales a $15{ }^{\circ} \mathrm{C}$; días cálidos (C), con máximas mayores a $15{ }^{\circ} \mathrm{C}$ y menores o iguales a $25{ }^{\circ} \mathrm{C}$ y, por último, los días muy cálidos (MC) que corresponden a aquellos cuyas temperaturas máximas superaron los $25^{\circ} \mathrm{C}$.

Finalmente, la precipitación se dividió en días lluviosos $(P)$ y días secos, según se registren o no precipitaciones.

Del análisis y consideración de las tres variables mencionadas según Pejenaute Goñi (1990), aparecen cinco grandes grupos de tipos de tiempo con cuatro tiempos cada uno:

- Tipos de tiempo muy fríos (MF): Anticiclón muy frío con precipitaciones (AMFP); Anticiclón muy frío sin precipitaciones (AMF); Ciclón muy frío con precipitaciones (CMFP) y Ciclón muy frío sin precipitaciones (CMF).

- Tipos de tiempo fríos (F): Anticiclón frío con precipitaciones (AFP); Anticiclón frío 
sin precipitaciones (AF); Ciclón frío con precipitaciones (CFP) y Ciclón frío sin precipitaciones (CF).

- Tipos de tiempo Templados (T): Anticiclón templado con precipitaciones (ATP); Anticiclón templado sin precipitaciones (AT); Ciclón templado con precipitaciones (CTP) y Ciclón templado sin precipitaciones $(\mathrm{CT})$.

- Tipos de tiempo Cálidos (C): Anticiclón cálido con precipitaciones (ACP); Anticiclón cálido sin precipitaciones (AC); Ciclón cálido con precipitaciones (CCP) y Ciclón cálido sin precipitaciones (CC).

- Tipos de tiempo Muy Cálidos (MC): Anticiclón muy cálido con precipitaciones (AMCP); Anticiclón muy cálido sin precipitaciones (AMC); Ciclón muy cálido con precipitaciones (CMCP) y Ciclón muy cálido sin precipitaciones (CMC).

Paralelamente se analizaron, a partir de las cartas de tiempo, las masas de aire que generaron los estados de tiempo (climatología dinámica). Es decir, se explicaron las situaciones atmosféricas, la posición de los centros de alta y baja presión y la trayectoria de las masas de aire. Las cartas de tiempo están expresadas en hora Zulu (Z), por ser cartas de navegación aérea y corresponde a la hora en la longitud $0^{\circ}$. Este sistema privilegia el uso de una hora común (Greenwich) sobre la hora local que implicaría conversiones para transformación horaria.

\section{Resultados y discusión}

El período considerado, 2000-2005, presentó una presión media de 1.013,8 hPa. Los años 2000, 2001 y 2004 tuvieron medias anuales por encima de dicho valor. La temperatura máxima diaria media fue de $16,9{ }^{\circ} \mathrm{C}$ y los años 2000 , 2001 y 2004 presentaron valores más bajos que su media. Con respecto a la precipitación el año más lluvioso fue el 2001, con 698,4 mm mientras que el más seco fue el año 2003, con 382,2 mm en todo el período. El año 2000 no se consideró ya que no se poseen datos pluviométricos completos (Cuadro $N^{\circ} 1$ ).
Cuadro $\mathrm{N}^{\circ} 1$

VALORES PROMEDIOS DE PRESIÓN Y TEMPERATURA MÁXIMA Y PRECIPITACIÓN TOTAL ANUAL DE LA CIUDAD DE PUNTA ALTA EN EL PERÍODO 2000 - 2005

\begin{tabular}{|cccc|}
\hline Año & Presión & T$^{\circ}$ Máx & Precipitación \\
\hline 2000 & $1.016,3$ & 13,7 & S/D completos \\
2001 & $1.015,9$ & 16,4 & 698,4 \\
2002 & $1.012,6$ & 17,3 & 604,0 \\
2003 & $1.012,1$ & 16,9 & 382,2 \\
2004 & $1.013,9$ & 16,4 & 608,4 \\
2005 & $1.011,9$ & 20,4 & 335,4 \\
& $1.013,8$ & 16,8 & \\
\hline
\end{tabular}

S/D: Sin datos.

Fuente: Elaboración propia.

Como se ha dicho, la investigación puso énfasis en el análisis del año 2003, se analizaron en él un total de 347 días, pues, por desperfectos técnicos la estación meteorológica no registró datos completos durante 18 días. El 2003 tuvo una presión media de $1.012,1 \mathrm{hPa}$, valor bajo respecto de la media total de referencia. La temperatura máxima diaria media fue de $16,9^{\circ} \mathrm{C}$. El año fue seco, se registraron $382,2 \mathrm{~mm}$ de precipitación. Estas oscilaciones en las lluvias son típicas del área de estudio ya que se encuentra en un área de transición pluviométrica.

En relación con los estados de tiempo acaecidos, predominaron los tiempos ciclónicos (216 días) sobre los anticiclónicos (131 días) y los cálidos y muy cálidos sobre los templados y fríos, con 277 y 70 días respectivamente. En ningún caso se presentaron días muy fríos con temperaturas máximas diarias menores a los $5{ }^{\circ} \mathrm{C}$. Los eventos de precipitación ocurrieron en jornadas ciclónicas las cuales sumaron 66 sobre 95 días de lluvias del año. Las jornadas con precipitaciones fueron con temperaturas altas, tiempos cálidos y muy cálidos.

Se analizó la información estacionalmente; se consideraron los datos del verano a partir del 21 de diciembre de 2002. Las restantes estaciones se fijaron a partir del 21 
de marzo, otoño; invierno a partir del 21 de junio y primavera del 21 de septiembre al 20 de diciembre de 2003.

\section{Verano}

La estación estival presentó una presión media de 1.007,2 hPa con una máxima de $1.016,8 \mathrm{hPa}$ el día 30 de diciembre y una mínima de 991,8 hPa el 19 de marzo. Los registros muestran una predominancia de los tiempos ciclónicos sobre los anticiclónicos. La temperatura máxima media fue de $29,4{ }^{\circ} \mathrm{C}$, con un valor máximo de $38,7^{\circ} \mathrm{C}$. La precipitación total ascendió a $80 \mathrm{~mm}$, siendo el día que más Ilovió el 12 de enero con 13,8 mm. El tiempo reinante fue ciclónico muy cálido el cual se desarrolló durante 10 días consecutivos.

El verano se caracterizó por una permanencia de tiempos ciclónicos cálidos y muy cálidos. Estos estados atmosféricos fueron a su vez los causantes de los 20 días de precipitación que se presentaron en todo el período. Durante las jornadas calurosas con temperaturas máximas oscilando entre 30 y $37{ }^{\circ} \mathrm{C}$ y con la presión en leve descenso con valores que pueden oscilar entre un máximo de $1.012 \mathrm{mb}$ y un mínimo de $998 \mathrm{mb}$, son propensas según Celemin (1984: 209) a la formación de las Ilamadas "tormentas de masa de aire". Los tiempos anticiclónicos solo sucedieron durante 9 días. La mayor frecuencia de tipo de tiempo fue el ciclónico muy cálido (CMC) con 57 días y representa en el conjunto el $64 \%$ del total.

La situación sinóptica que ejemplifica el estado de tiempo dominante para el verano (CMC) se presenta en la Figura $N^{\circ} 2$ correspondiente a la carta de tiempo del día 10 de marzo (1200 Z). En la misma carta se observan el anticiclón del Atlántico Sur y el del Pacífico Sur desplazados hacia el Sur como consecuencia del movimiento aparente del Sol. Desde los $40^{\circ}$ de latitud hacia el Sur predomina el cinturón de las Bajas Presiones Subpolares que afecta a la Patagonia argentina. Por otra parte, se distingue en el centro Norte de la Argentina un área dominada por bajas presiones de origen térmico, con valores inferiores a $1.005 \mathrm{hPa}$ que interrumpen el cinturón de las Altas Presiones Subtropicales.
Se aprecia también un frente frío con trayectoria Suroeste - Noreste. En las provincias de Buenos Aires y La Pampa comienzan las condiciones propicias para la formación de una línea de estabilidad. El sistema de mal tiempo afecta todo el centro - Este del país con precipitación y áreas de nieblas. La presión en el área de estudio oscila entre los $1.003,5$ hPa y los 1.005,3 hPa; el cielo se encuentra nublado en toda la costa bonaerense y en el Sureste de la provincia se observan precipitaciones. Las temperaturas máximas diarias varían entre los $21^{\circ} \mathrm{C}$ y $24^{\circ} \mathrm{C}$, y las temperaturas del punto de rocío tienen valores muy próximos a las del aire lo cual favorece la formación de nieblas.

\section{Otoño}

La estación otoñal tuvo una presión media de 1.013,8 hPa, con una máxima de 1.027,7 hPa y una mínima de 1.000,6 hPa, ocurridas los días 18 de mayo y 8 de abril respectivamente. Los registros no muestran marcadas diferencias entre los días con tiempos ciclónicos y los anticiclónicos. La temperatura máxima diaria media fue de $18,7^{\circ} \mathrm{C}$, siendo el mayor valor $31,7^{\circ} \mathrm{C}$. Los días con precipitación fueron 33, totalizando $94,4 \mathrm{~mm}$. La mayor intensidad diaria fue de 33,2 $\mathrm{mm}$ el 14 de mayo y se presentó con tiempo ciclónico cálido (CCP).

Los estados de tiempo tuvieron mayor persistencia pero se presentaron mucho más variados. Con respecto a la presión, los días ciclónicos superaron levemente a los anticiclónicos mientras que la temperatura presentó mayor diferenciación. Los datos muestran nuevamente el predominio de los tiempos cálidos (63 días), le siguen los templados (19 días) y los muy cálidos (10 días). No se observan temperaturas máximas diarias frías o muy frías. Las precipitaciones se presentaron tanto en tiempos ciclónicos (19 días) como anticiclónicos (14 días) y de todos ellos 25 se dieron con temperaturas máximas diarias superiores a los $15{ }^{\circ} \mathrm{C}$. La mayor frecuencia de tiempo fue el ciclónico cálido (CC), con 20 días y representa en el conjunto el $21,7 \%$ del total.

La carta sinóptica del 18 de abril (1.200 Z) ejemplifica las condiciones que caracterizaron a los días ciclónicos cálidos (CC). En 
Figura $\mathrm{N}^{\circ} 2$

ESTADO DE TIEMPO DOMINANTE PARA EL VERANO (CMC).

CARTA DEL TIEMPO DEL 10 DE MARZO DE 2003, 1200 Z. AMPLIACIÓN PROVINCIA DE BUENOS AIRES

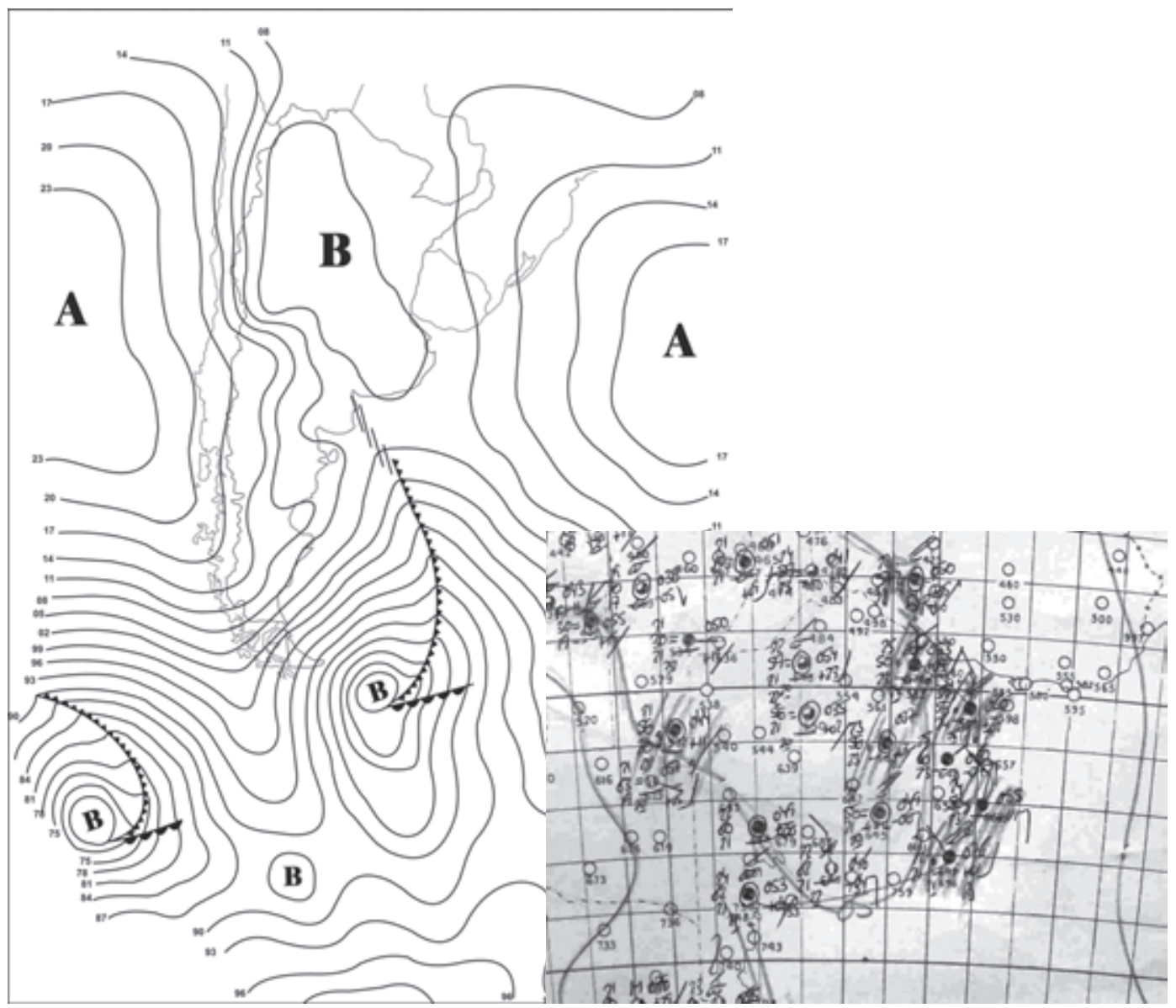

Fuente: Servicio Meteorológico Nacional (2003).

ella se observan los anticiclones semipermantes del Pacífico Sur y del Atlántico Sur, aproximadamente sobre los $30^{\circ}$ de latitud Sur (Figura $\mathrm{N}^{\circ} 3$ ). El primero de ellos ejerce su influencia en todo el sector Oeste y región patagónica del país con un valor central de $1.023 \mathrm{hPa}$. Por otra parte, sobre el Noreste de la provincia de Buenos Aires se ubica un centro de baja presión de $999 \mathrm{hPa}$ (onda frontal) desde cual sale hacia el Norte un frente frío y hacia el Este una rama cálida. Las precipitaciones se extienden en gran parte de la región pampeana y Noreste argentino. Sobre la costa bonaerense soplan vientos del Sureste entre 15 a 20 nudos. En la región patagónica se introduce un sistema de alta presión asociado a aire frío. Las estaciones meteorológicas del área de estudio muestran cielos totalmente cubiertos con nubes de tipo altocúmulos y altostratus. Cabe destacar que este estado de tiempo es típico del litoral bonaerense y se reconoce como Sudestada.

\section{Invierno}

El invierno tuvo una presión media de $1.018,5 \mathrm{hPa}$, con una máxima de 1.031,6 hPa el día 13 de septiembre y una mínima de $1.002,9 \mathrm{hPa}$ el 7 de julio. El va- 
Figura $\mathrm{N}^{\circ} 3$

SITUACIÓN ESTADO DE TIEMPO DOMINANTE PARA EL OTOÑO (CC).

CARTA DEL TIEMPO DEL 18 DE ABRIL DE 2003,1200 Z. AMPLIACIÓN PROVINCIA DE BUENOS AIRES

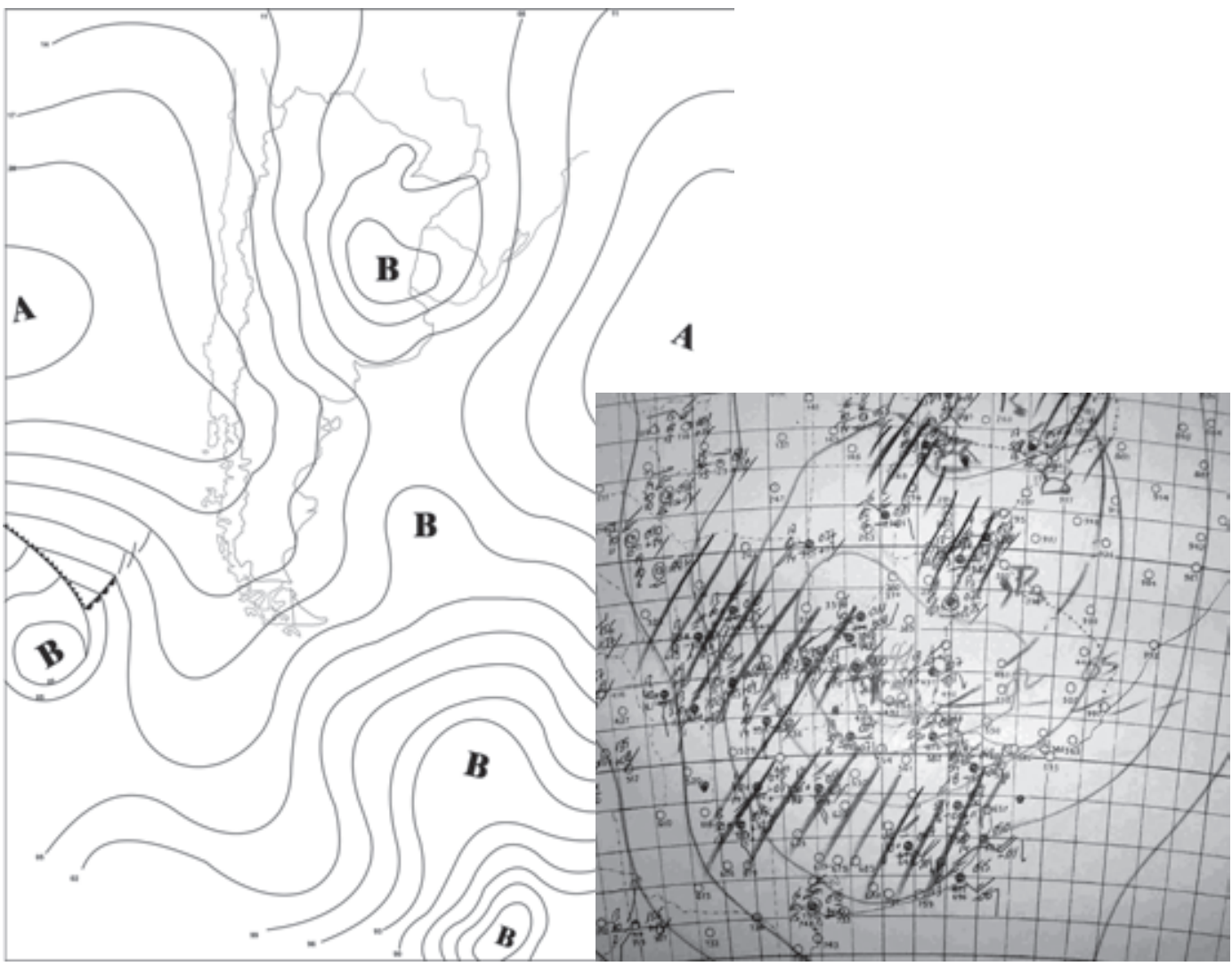

Fuente: Servicio Meteorológico Nacional (2003).

lor máximo mencionado coincide a su vez, con el registro más alto de presión de todo el 2003. Predominaron los tiempos anticiclónicos con 54 días sobre los ciclónicos con 20 días. La temperatura máxima diaria media fue de $14,5{ }^{\circ} \mathrm{C}$ y el mayor valor de $24,7{ }^{\circ} \mathrm{C}$ se presentó con tiempo ciclónico luego de 4 días con temperaturas máximas por encima de los $20{ }^{\circ} \mathrm{C}$. La precipitación total fue de $27,6 \mathrm{~mm}$ y la máxima se produjo el 27 de junio, con 7,8 mm. Si bien se registraron lluvias durante 18 días, las mismas fueron con montos insignificantes en su gran mayoría y se presentaron tanto con tiempo ciclónico como anticiclónico.

En el invierno, el estado de tiempo que predominó fue el anticiclón templado (AT) que representó 36 días del total $(48,6 \%)$ de los cuales 8 se presentaron con precipitación. Los días templados también se presentaron con tiempo ciclónico (CT) aunque con mucha menor frecuencia. Se observan a su vez, 26 días donde la temperatura máxima diaria superó los $15^{\circ} \mathrm{C}$ y 3 en que la temperatura máxima diaria estuvo entre los $5^{\circ} \mathrm{C}$ y los $10^{\circ} \mathrm{C}$. No se observaron días muy fríos ni muy cálidos.

La carta sinóptica del día 26 de junio (1.200 Z) es un buen ejemplo del estado de tiempo predominante (AT). En ella se observan las Altas Presiones Subtropicales situadas en los océanos entre las latitudes de $15^{\circ}$ Sur y $25^{\circ}$ Sur (Figura $N^{\circ} 4$ ). Por otra parte, una zona de baja presión se ubica sobre Bolivia y afecta al Noroeste de la Argentina. A los $35^{\circ}$ de latitud Sur, en el océano Pacífico 

AMPLIACIÓN PROVINCIA DE BUENOS AIRES

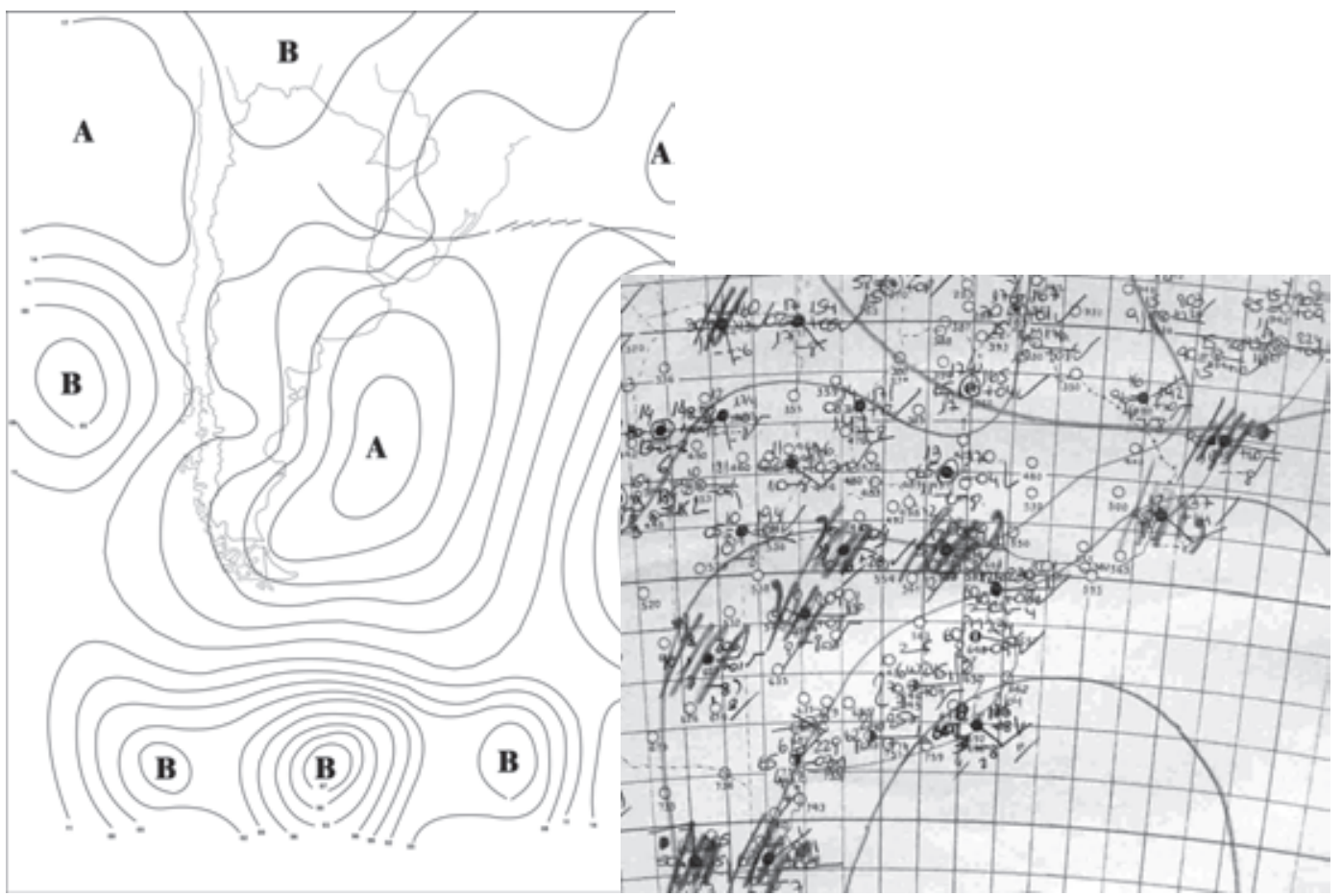

Fuente: Servicio Meteorológico Nacional (2003).

se ubica un centro de baja presión con valores de $1.005 \mathrm{hPa}$. Gran parte del país se encuentra bajo la influencia de un anticiclón $(1.029 \mathrm{hPa})$ situado sobre las costas de la Patagonia y ocasiona lluvias en todo su litoral. En el área de estudio, con valores de presión entre 1.022 y $1.025 \mathrm{hPa}$, se observan cielos parcial o totalmente cubiertos pero sin registrarse precipitaciones. Los vientos soplan del Este y Sureste con muy poca intensidad.

\section{Primavera}

En la estación primaveral se analizaron 92 días de los cuales 68 correspondieron a tiempos ciclónicos y 24 a anticiclónicos. La presión media de la estación fue $1.010,1$ hPa con una máxima de $1.025,9 \mathrm{hPa}$ el 1 de noviembre y una mínima de 998,7 hPa el 10 de diciembre. La temperatura máxima diaria media fue de
$24,5{ }^{\circ} \mathrm{C}$, registrándose el máximo valor el 18 de diciembre $\left(36,4{ }^{\circ} \mathrm{C}\right)$ con estado de tiempo ciclónico $(\mathrm{CC})$. Los días con precipitación fueron 22, de los cuales 15 correspondieron a tiempo ciclónico cálido (CCP). A diferencia de la estación invernal, las Iluvias en esta estación fueron más abundantes ya que en total ascendieron a $172,6 \mathrm{~mm}$. El máximo valor pluviométrico fue $21 \mathrm{~mm}$ y se produjo con un tiempo AT el 25 de septiembre, como consecuencia de un avance frontal.

Los tiempos cálidos (C) fueron los que dominaron 49 días y los muy cálidos (MC) 40 , lo que significa que de los 92 días analizados 89 tuvieron temperaturas máximas diarias mayores a los $15^{\circ} \mathrm{C}$. No se registraron días fríos o muy fríos. El estado de tiempo predominante fue el ciclónico muy cálido (CMC), con 27 días del total, lo que representa el $29,3 \%$. 
La carta sinóptica del día 7 de noviembre (1.200 Z) ejemplifica la situación atmosférica reinante durante un tiempo ciclónico muy cálido (CMC). En la misma se observan los centros de Altas Presiones Subtropicales situados sobre los océanos con valores de $1.017 \mathrm{hPa}$ (Figura $\mathrm{N}^{\circ}$ 5). Por otra parte, se observa un centro de baja presión de 1.005 hPa en el centro Oeste de la Argentina unido mediante una onda frontal al cinturón de Bajas Presiones Subpolares que afecta todo el sector patagónico. En el área de estudio, pese a haber ocurrido el pasaje del frente frío, persistieron las temperaturas elevadas $\left(22^{\circ} \mathrm{C}\right)$ y la presión, al contrario, fue baja $(1.006,4 \mathrm{hPa})$. El cielo estuvo parcialmente nublado con vientos del Suroeste. Todo el centro de la provincia de Buenos Aires presentó alta temperaturas.

\section{Consideraciones finales}

El método utilizado permitió analizar en forma detallada los estados de tiempo y las condiciones atmosféricas reinantes en el Suroeste bonaerense y específicamente para la ciudad de Punta Alta. Se observó un predominio de tiempos ciclónicos muy cálidos, especialmente en verano y primavera. Los tiempos ciclónicos cálidos (CC) estuvieron presentes especialmente en las temporadas otoñales y primaverales. Los tiempos anticiclónicos templados (AT) dominaron en la estación invernal. Por otra parte, los estados de tiempo muy fríos (AMFP, AMF, CMFP y CMF) y ciclónicos fríos (CF, CFP) están totalmente ausentes (Cuadro $\mathrm{N}^{\circ} 2$ ).

Figura $\mathrm{N}^{\circ} 5$

SITUACIÓN ESTADO DE TIEMPO DOMINANTE PARA LA PRIMAVERA (CMC). CARTA DEL TIEMPO DEL 7 DE NOVIEMBRE DE 2003, 1.200 Z. AMPLIACIÓN PROVINCIA DE BUENOS AIRES

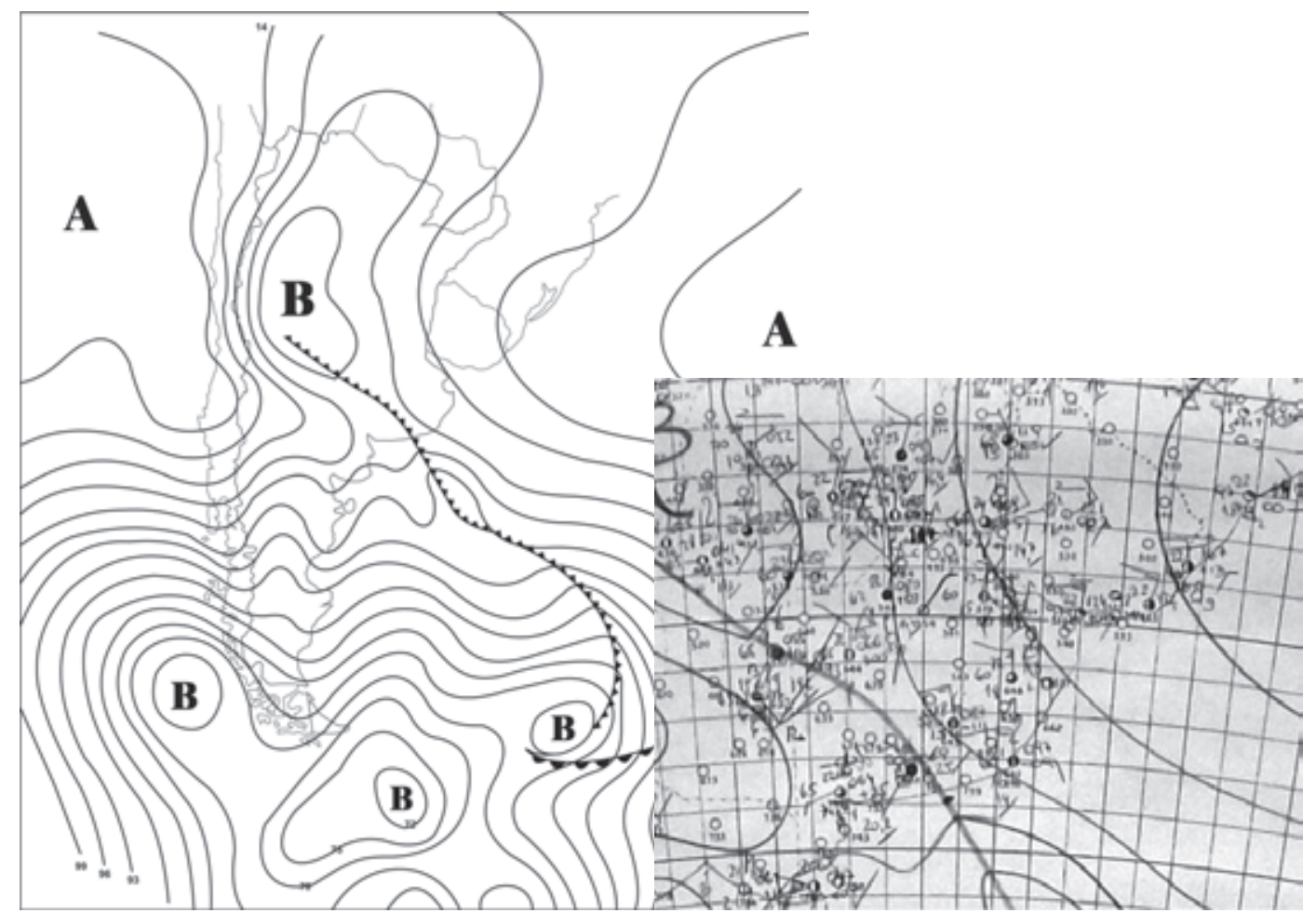

Fuente: Servicio Meteorológico Nacional (2003). 
Cuadro $\mathrm{N}^{\circ} 2$

FRECUENCIA EN DÍAS Y PORCENTAJE DE ESTADOS DE TIEMPO PARA EL AÑO 2003. NOMENCLATURA DE ESTADOS DE TIEMPO EXPLICADAS EN EL TEXTO

\begin{tabular}{|c|c|c|c|c|c|c|}
\hline \multirow{2}{*}{$\begin{array}{l}\text { Estado de } \\
\text { Tiempo }\end{array}$} & \multicolumn{2}{|c|}{ Anual } & \multirow{2}{*}{$\begin{array}{l}\text { Verano } \\
\text { Días }\end{array}$} & \multirow{2}{*}{$\begin{array}{l}\text { Otoño } \\
\text { Días }\end{array}$} & \multirow{2}{*}{$\begin{array}{c}\text { Invierno } \\
\text { Días }\end{array}$} & \multirow{2}{*}{$\begin{array}{c}\text { Primavera } \\
\text { Días }\end{array}$} \\
\hline & Días & $\%$ & & & & \\
\hline AMFP & 0 & 0,0 & & & & \\
\hline AMF & 0 & 0,0 & & & & \\
\hline CMFP & 0 & 0,0 & & & & \\
\hline CMF & 0 & 0,0 & & & & \\
\hline CFP & 0 & 0,0 & & & & \\
\hline $\mathrm{CF}$ & 0 & 0,0 & & & & \\
\hline AFP & 1 & 0,3 & & & 1 & \\
\hline $\mathrm{AF}$ & 2 & 0,6 & & & 2 & \\
\hline AMCP & 2 & 0,6 & & & & 2 \\
\hline CT & 6 & 1,7 & & 3 & 3 & \\
\hline СТP & 11 & 3,2 & & 5 & 6 & \\
\hline ATP & 13 & 3,7 & & 3 & 8 & 2 \\
\hline $\mathrm{ACP}$ & 13 & 3,7 & & 11 & 1 & 1 \\
\hline AMC & 15 & 4,3 & 5 & 3 & & 7 \\
\hline СМСР & 20 & 5,8 & 15 & 1 & & 4 \\
\hline ССР & 35 & 10,1 & 5 & 13 & 2 & 15 \\
\hline AT & 37 & 10,7 & & 8 & 28 & 1 \\
\hline AC & 48 & 13,8 & 4 & 19 & 14 & 11 \\
\hline CC & 54 & 15,6 & 3 & 20 & 9 & 22 \\
\hline CMC & 90 & 25,9 & 57 & 6 & & 27 \\
\hline TOTAL & 347 & 100,0 & 89 & 92 & 74 & 92 \\
\hline
\end{tabular}

Fuente: Elaboración propia.

El análisis de cada una de las variables permite concluir que los tiempos ciclónicos predominaron en 2003 en verano y primavera. El invierno fue la única estación del año con dominio de tiempo anticiclónico. Con respecto a la temperatura, los tiempos muy cálidos se dieron en 127 días con predominio en verano y ausencia total en invierno. Los días cálidos, es decir, con temperaturas máximas diarias entre $15{ }^{\circ} \mathrm{C}$ y $25^{\circ} \mathrm{C}$, se registraron todo el año, especialmente en otoño y primavera. Los templados dominaron durante invierno y los tiempos fríos solo se presentaron durante 3 días en la estación invernal. No hubo días con máximas diarias inferiores a $5{ }^{\circ} \mathrm{C}$ en todo 2003 (MF). Por último, las precipitaciones se registraron durante 95 días, con mayor ocurrencia e intensidad en otoño pero con mayor cantidad en primavera. La estación más seca fue el invierno, que presentó la menor pluviosidad y la menor cantidad de días con lluvias (Cuadro $\mathrm{N}^{\circ} 3$ ).

Las cartas del tiempo constituyeron una herramienta fundamental e indispensable en el análisis de los estados de tiempo, ya que permitieron explicar las situaciones atmosféricas que produjeron los estados dominantes en cada estación en especial la posición de los centros de acción, frentes fríos y cálidos y las áreas efectuadas por avances de zonas de inestabilidad. 
Cuadro $\mathrm{N}^{\circ} 3$

FRECUENCIA EN DÍAS Y PORCENTAJE DE LOS ESTADOS DE TIEMPO SEGÚN ELEMENTOS CLIMÁTICOS PARA EL AÑO 2003

\begin{tabular}{|c|c|c|c|c|c|c|}
\hline \multirow{2}{*}{$\begin{array}{c}\text { Estado de } \\
\text { Tiempo }\end{array}$} & \multicolumn{2}{|c|}{ Anual } & \multirow{2}{*}{$\begin{array}{l}\text { Verano } \\
\text { Días }\end{array}$} & \multirow{2}{*}{$\begin{array}{l}\text { Otoño } \\
\text { Días }\end{array}$} & \multirow{2}{*}{$\begin{array}{l}\text { Invierno } \\
\text { Días }\end{array}$} & \multirow{2}{*}{$\begin{array}{c}\text { Primavera } \\
\text { Días }\end{array}$} \\
\hline & Días & $\%$ & & & & \\
\hline Anticiclón & 131 & 37,8 & 9 & 44 & 54 & 24 \\
\hline Ciclón & 216 & 62,2 & 80 & 48 & 20 & 68 \\
\hline Total & 347 & 100,0 & 89 & 92 & 74 & 92 \\
\hline Muy Fríos & 0 & 0,0 & 0 & 0 & 0 & 0 \\
\hline Fríos & 3 & 0,9 & 0 & 0 & 3 & 0 \\
\hline Templados & 67 & 19,3 & 0 & 19 & 45 & 3 \\
\hline Cálidos & 150 & 43,2 & 12 & 63 & 26 & 49 \\
\hline Muy Cálidos & 127 & 36,6 & 77 & 10 & 0 & 40 \\
\hline Total & 347 & 100,0 & 89 & 92 & 74 & 92 \\
\hline Con Precipitación & 95 & 27,4 & 20 & 33 & 18 & 24 \\
\hline Sin Precipitación & 252 & 72,6 & 69 & 59 & 56 & 68 \\
\hline Total & 347 & 100,0 & 89 & 92 & 74 & 92 \\
\hline
\end{tabular}

Fuente: Elaboración propia.

Cabe destacar actualmente se están realizando de forma paralela estudios biometeorológicos dentro del marco del Proyecto de Investigación en el que se desarrolla este trabajo. En particular se analizan las posibles relaciones entre las enfermedades que la población padece y los diferentes estados de tiempo. Para lograr estos objetivos es fundamental realizar previamente estudios pormenorizados para caracterizar y categorizar las situaciones sinópticas dominantes y recurrentes en el Suroeste bonaerense.

\section{Referencias bibliográficas}

ABAURREA, J.; ÁlVAREZ, E.; ASÍN, J.; CEBRIÁN, A. C. y CENTELLES, A. Situaciones sinópticas más frecuentes en los episodios de ola de calor en el centro del Valle del Ebro. Zaragoza: Dpto. de Métodos Estadísticos, Univ. de Zaragoza Centro Meteorológico Territorial del INM en Zaragoza Asociación Meteorológica Española, s/f.

ALMARZA, C. Las estadísticas del "tiempo". Índice: Revista de Estadísticas y Sociedad, 2005, No 10, p. 15-17.
BARTH, H \& STEINKOHL, F. Origin of winter precipitation in the central coastal lowlands of Saudi Arabia. Journal of Arid Environments, 2004, № 57, p. 101-115.

CAMPO DE FERRERAS, A.; CAPELLI DE STEFFENS, A. y DIEZ, P. El clima del Suroeste Bonaerense. Bahía Blanca: Departamento de Geografía y Turismo. Universidad Nacional del Sur, 2004.

CAPELLI DE STEFFENS, A.; PICCOLO, M. y CAMPO DE FERRERAS, A. Clima urbano de Bahía Blanca. Bahía Blanca: Departamento de Geografía y Turismo: Universidad Nacional del Sur - Editorial Dunken, 2005.

CAVIEDES C. Los estados de tiempo típicos de Valparaíso, Chile Central. Revista Instituto de Historia y Geografía de la Universidad Católica de Valparaíso, 1969, № 3.

CELEMIN, A. H. Meteorología Práctica. Buenos Aires: Ed. Mar del Plata, 1984.

FONT TUlLOT, I. Climatología de España y Portugal. Madrid: Instituto Nacional de Meteorología. Madrid, 2000, p. 111-144. 
LAM, נ.; TSANG, C.; YANG, L. y LI, D. Weather data analysis and design implications for different climatic zones in China. Building and Environment, 2005, No 40, p. 277-296.

MARTÍN VIDE, J. Mapas del tiempo: fundamentos, interpretación e imágenes de satélite. Barcelona: Oikos Tau, 1991.

MARTÍN VIDE, J. y OLCINA CANTOS, J. Climas y tiempos de España. Madrid: Alianza Editorial, 2001.

OLGYAY, V. Design with climate-bioclimatic approach to architectural regionalism. New York: Van Nostrand Reinhold, 1992.

PEDELABORDE, P. Introdution a I'Etude Scientifique du Climat. Paris: Société d'Edition d'Enseignement Supérieur, 1970.

PEJENAUTE GOÑI, J. Tipos de tiempo y clima de las comarcas Navarras. Pamplona:
Gobierno de Navarra - Departamento de Educación, Cultura y Deporte, 1990.

REY BENADERO, F. Los conocimientos geográficos en la predicción de fenómenos meteorológicos de rango extraordinario. Boletín de la A.G.E., 2001, $\mathrm{N}^{\circ}$ 31, p. 195-204.

RIVERA, A. Las situaciones de Iluvias torrenciales en el área mediterránea española y el Plan PREVIMET. Revista del Aficionado a la Meteorología, 2003, № 7.

SERVICIO METEOROLÓGICO NACIONAL. Masas de aire y frentes. Boletín Informativo No 31. Buenos Aires: Servicio Meteorológico Nacional, 1987.

SERVICIO METEOROLÓGICO NACIONAL. Cartas del tiempo año 2003, 1200 Z. Ampliación provincia de Buenos Aires. Buenos Aires: Servicio Meteorológico Nacional, 2003. 
